Available online at www.sciencedirect.com science 0 directo

\section{ANNALS \\ OF ANATOMY \\ www.elsevier.de/aanat}

\title{
The collagenic structure of human digital skin seen by scanning electron microscopy after Ohtani maceration technique
}

\author{
Simone Sangiorgi $i^{a, *}$, Alessandro Manelli ${ }^{a}$, Marina Protasoni ${ }^{a}$, \\ Mario Ronga ${ }^{\mathrm{b}}$, Mario Raspanti ${ }^{\mathrm{a}}$
}

\author{
aDepartment of Human Morphology, Laboratory of Morphology "L. Cattaneo", University of Insubria, Via Monte \\ Generoso, 71, 21100 Varese, Italy \\ ${ }^{\mathrm{b}}$ Department of Orthopedic and Traumatologic Sciences, University of Insubria, Viale Borri 57, Varese, Italy
}

Received 3 May 2004; accepted 4 June 2004

\section{KEYWORDS \\ Scanning electron microscope; \\ Othani maceration; \\ Collagen; \\ Human toe; \\ Skin}

\begin{abstract}
Summary
We performed a morphological scanning electron microscope (SEM) study to describe the fine structure and disposition of collagenous tissue in the human toe.

After therapeutic amputation of a human right leg, we applied the Othani maceration technique to the skin of three toes surgically explanted from the foot.

We distinguished eight cutaneous regions and focused on some specialized collagenous structures differing in the thickness of the skin. The eight areas investigated were: the dorsal skin, the eponychium, the perionychium, the hyponychium, the region under the visible nail, the nail root, the plantar skin and finally the toe tip. Each of these areas is characterized by a distinctive collagenous surface disposition, with some peculiar features mostly related to dermal papillae. At high magnification, we observed the spatial arrangement of the collagen fibers constituting the top of the dermal papillae that represents the attachment site of the proliferative basal layer of the epidermis. We also noted an impressive density of collagen fibers throughout the thickness of the dermal layer, organized in specialized structures and constituting the skeleton of dermal thermoreceptorial corpuscles or sweat glands.

A combination of SEM and Ohtani technique disclosed the three-dimensional architecture of the collagenous matrix of tarsal skin under physiologic conditions, giving a detailed description of the most reactive tissue during pathologic processes. (c) 2005 Elsevier $\mathrm{GmbH}$. All rights reserved.
\end{abstract}

*Corresponding author. Tel.: +1 390332 264847; fax: +1 390332263324

E-mail address: simo.med@libero.it (S. Sangiorgi). 


\section{Introduction}

The collagenous structure of the human digit plays an important role in a number of pathological processes mainly comprising the changes occurring related to traumatic injury, ischemic processes, diseases involving collagen synthesis dysfunction and other metabolic disorders affecting the extremities such as diabetes (Wilborn and Montes, 1979; Torre and Varetto, 1985; Torre et al., 1986; Warfel and Hull, 1989; Moore et al., 1993; Vaccaro et al., 1999).

The production of collagen fibers strictly depends on the activity of fibroblasts and the local production of many growth factors influencing their activity (Reed and lozzo, 2002).

Morphological studies were then aimed at finding a specific maceration technique to yield the clearest possible three-dimensional demonstration of collagenous tissue.

The first method used to study the dermalepidermal junction was described by Scaletta and MacCullum and consisted in a first treatment in a balanced salt solution containing EDTA and then an incubation with trypsin at $37^{\circ} \mathrm{C}$ (Kitano and Okada, 1983).

The persistence of the remaining basal cells in some cases proved troublesome and sometimes the collagenous structure was covered by cellular cytoplasmic extensions. By contrast, strong alkali treatment at low concentrations allowed the digestion of all organic tissues except collagen fibers: in particular, the Ohtani maceration protocol consists of a long fixation in aldehydes and subsequent digestion in $\mathrm{NaOH} 1 \mathrm{~N}$ solution (Ohtani and Ohtani, 2000) .

A combination of scanning electron microscope (SEM) investigation techniques and the Ohtani maceration method shed more light on the threedimensional disposition of collagenous fibers in many sites of the body.

We applied this method to human digital skin, describing its "organic skeleton" both in dermal and hypodermal layers.

Thanks to this technique it has been possible to retain only the collagen bundles that form the structure covering the human digit in their correct three-dimensional disposition without interferences due to other surrounding tissues: looking at the collagenous matrix we are looking at the skeleton of an organ and its structural elements (Tsuji, 1980; Tsugane and Yasuda, 1995; Yamada et al., 1996; Bolanowski and Pawson, 2003).

Moreover, we noticed the exact correspondence existing between the disposition of dermal papillae and the microvascular architecture of the human digit as described in our previous article (Sangiorgi et al., 2004).

Even if the collagenous structure of human digits plays an active part in many pathophysiological conditions, an in-depth morphological description is still lacking.

The aim of this study has been to draw a detailed map of the disposition of dermal papillae and the collagenous specialized structure sited in the human digital skin to highlight the structural features of their architecture.

We analyzed the shape and directions of dermal papillae in eight different regions trying to correlate their morphological shape with the physiological functions of the region itself.

This, in turn, is a starting point for better understanding the modifications occurring to the nail or digital skin in the course of many pathologic processes.

\section{Material and methods}

The right leg of a 50-year-old man was therapeutically explanted to prevent spread of a neoplastic mass located in the tibial bone. Soon after amputation, the entire leg was transported in an iced envelope to our anatomical laboratory where we amputated the second, third and fourth digits.

None of the digits had any macroscopic injury or visible pathologic processes and the nails were eutrophic and defined in shape. In addition, history taking had failed to disclose any trauma to the digits, systemic diseases or any other hereditary collagenous dysfunctions.

We isolated the vascular peduncle of each digit perfusing them through a $24 \mathrm{G}$ cannule, at first with heparin and then with Karnovsky fixative solution (0.25\% glutaraldehyde and $0.25 \%$ paraformaldehyde in $0.1 \mathrm{M} \mathrm{Na}$-cacodylate buffer at $\mathrm{pH}=7.2$ ).

The skin of each digit was then explanted under a dissecting stereomicroscope with care taken not to damage any superficial structure with forceps, and placed in the same fixative solution used in the previous perfusion procedure. The specimens were maintained in fixative for 2 weeks: the solution was changed regularly three times.

After the fixation process, each specimen was reduced with a razor blade and for each one we obtained two specimens, one from the dorsal region, and the other from the palmar region. All six skin specimens were then immersed in a solution of $\mathrm{NaOH} 1 \mathrm{~N}$ with $5 \%$ mycostatin to prevent fungal growth. The solution was changed daily to wash out the digested organic tissue. 
All the specimens were dehydrated in graded alcohol, critical point dried in an Emitech K850 CPD apparatus, mounted on aluminium stubs on adhesive film and coated with $10 \mathrm{~nm}$ of gold in an Emitech K250 sputter coater. Due to their size, some specimens needed metallic bridges to maintain good conduction all over the stub. The specimens were then observed in a Philips XL-30 FEG SEM at $10 \mathrm{kV}$.

\section{Results}

We organized the description of the specialized collagenous structures sited in the human digit dividing its entire surface into seven specific superficial areas and then, going deeper in the thickness of the skin, we also highlighted the features of dermal and hypodermal structures.

Looking at the superficial area of digital skin we can distinguish: the dorsal skin, the eponychium, the area under the visible nail, the hyponychium, the finger toe, the nail root, the perionychium and the plantar skin, represented graphically in the schematic drawing (Fig. 1).

\section{The dorsal skin}

At low magnification, the irregular disposition of dermal papillae is visibly characterized by a high variability both in shape and size without a preferential apparent orientation in the dorsal skin of the digit.

The papillae measure about $60-70 \mu \mathrm{m}$ in diameter and are $100 \mu \mathrm{m}$ high: the diameter at the

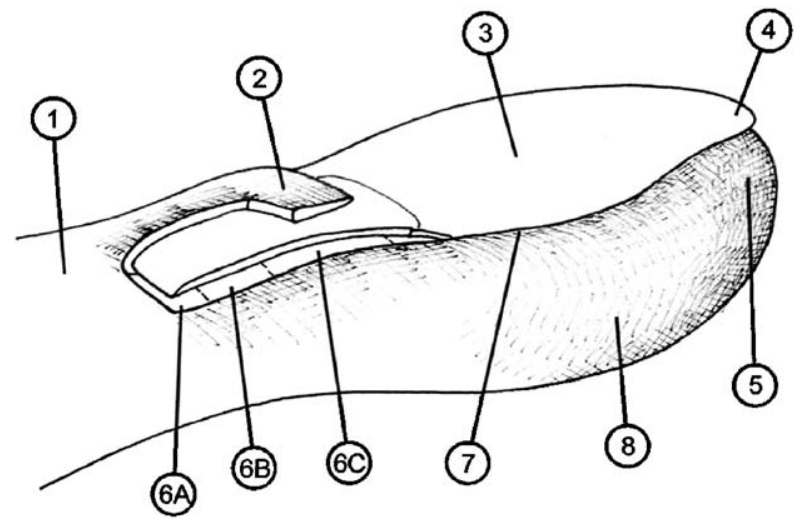

Figure 1. Graphic representation of a human digit: the labelled regions correspond to those described in the results: dorsal skin (1), eponychium (2), the area under the visible nail (3), the hyponychium (4), the digital tip (5), the nail root (6) divided into three parts: proximal $(6 \mathrm{~A})$, median (6B) and distal (6C), the perionychium (7) and the plantar skin (8). base of the papilla is similar to that measured at the top of it (Fig. 2A). Some dermal papillae are clustered into islets surrounded by deep grooves which may enclose the opening of the tubule of sweat glands and hair follicles.

Moving distally towards the eponychium, the height of the dermal papillae gradually decreases and the axes of the papillae lean toward the axis of the digit, following the natural growth direction of the nail.

At high magnification, many small holes can be seen at the bottom of each papilla representing the exit site of nerve fibers freely distributed inside the epidermis (Fig. 2B). Looking at the surface of
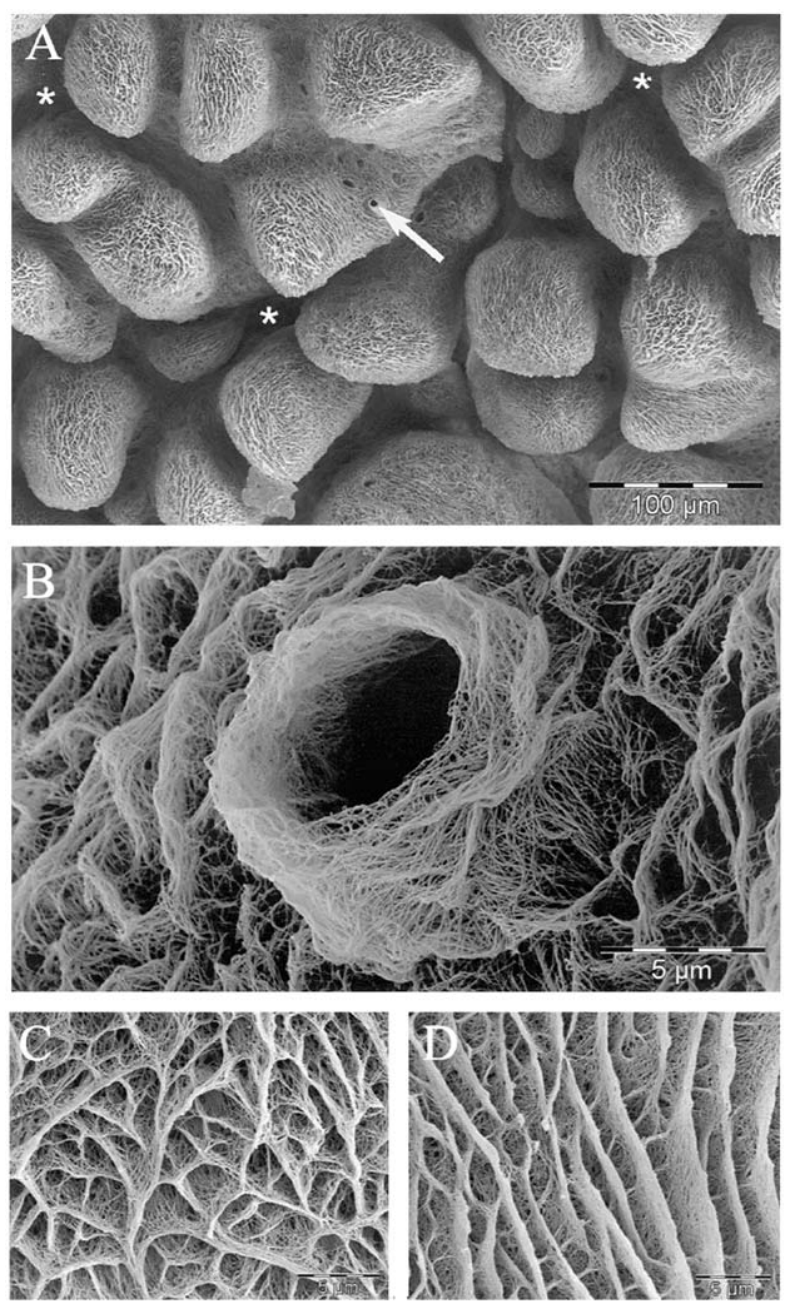

Figure 2. Dorsal skin area: dermal papillae organized in an islet $\left({ }^{*}\right)$ : note the small holes visible at the origin of each papillae demonstrating the presence of free nervous fibers entering the epidermal layer (arrow) (A). High magnification of a dermal papilla: we can observe the openings usually containing free nerve fibers (B) and two different patterns of collagen bundles on the papillary surface: regularly oriented $(\mathrm{C})$ and following an anastomotic drawing (D). 
the dermal papillae, there is a characteristic plexiform architecture made up of collagen bundles that can follow a preferential direction or create irregular tension (Fig. 2C/D).

\section{The eponychium}

The dermal papillae of the eponychium are oriented along the major axis of the digit and are $20-30 \mu \mathrm{m}$ high and about $20 \mu \mathrm{m}$ in diameter (Fig. 3A).

In the area closest to the nail, the dermal papillae are stretched and create a free margin
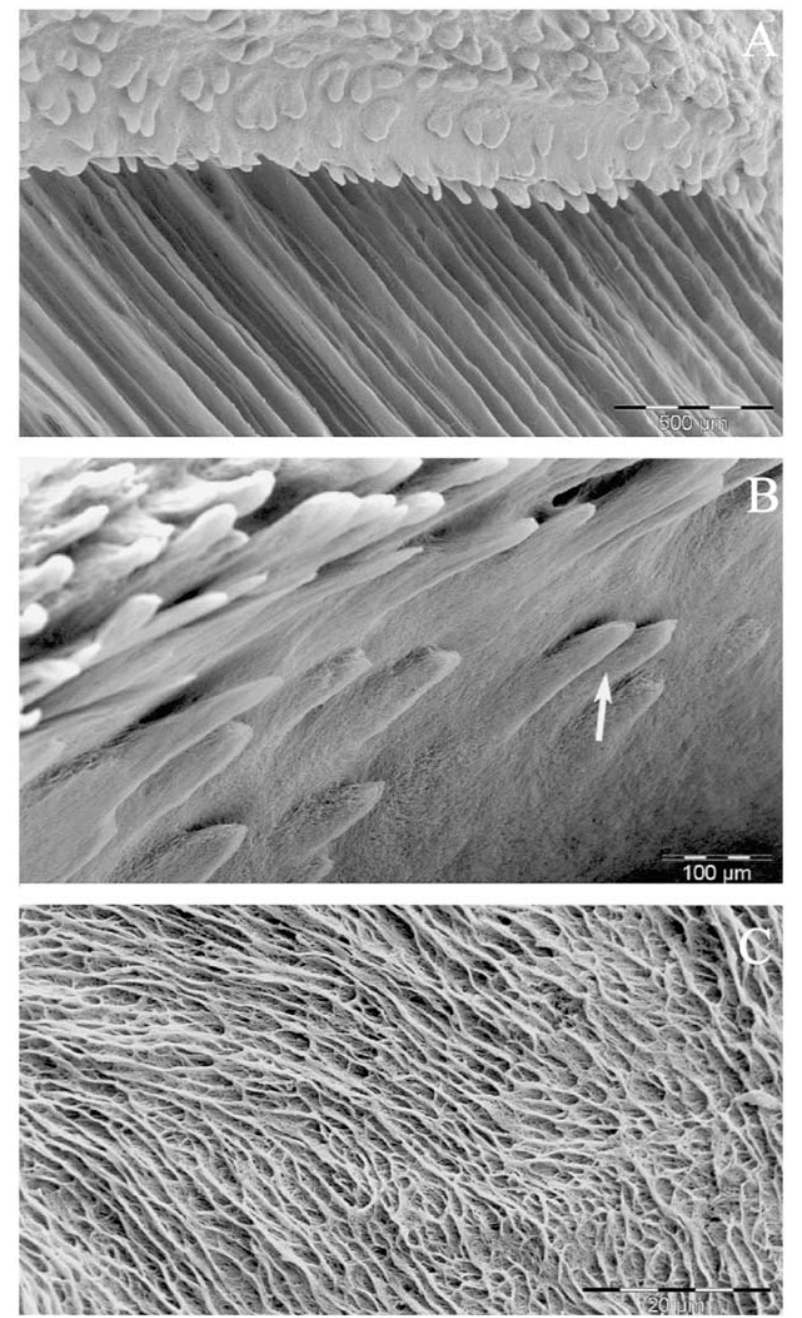

Figure 3. The eponychium: the free margin of the eponychium is constituted by stretched dermal papillae gradually reaching the point of invagination of the dorsal skin where we find three or four sheets of elongated papillae (A). The region under the eponychium, in close contact with the nail plate: we can clearly see some elongated dermal papillae (arrow) (B) and, at high magnification $(C)$, the smooth collagenic surface covering the nail plate to the nail matrix. in the lunula: when the skin of the eponychium is reflected and makes contact with the nail surface, its collagenic matrix assumes a stratified disposition made up of three-four layers of dermal papillae longitudinally oriented and elongated along the direction of nail growth (Fig. 3B).

These dermal papillae are clearly visible when we look at the region under the eponychium, in close contact with the nail: we no longer observe the exit holes of glands or the small holes of free intraepidermal nerve fibers.

Going along this surface towards the nail root, the dermal organization in papillae disappears, forming a wide, well-defined collagenous sheet with no visible specialized structures (Fig. $3 \mathrm{C}$ ).

\section{The area under the visible nail}

The collagenous matrix of the nail bed corresponding to the visible nail is made up of longitudinal parallel trabeculae, oriented along the major axis of the digit (Fig. 4A). At the top of these crestae, an irregular free margin is characterized by curved collagenous loops that sometimes form deep grooves inside the trabeculae itself (Fig. 4B).
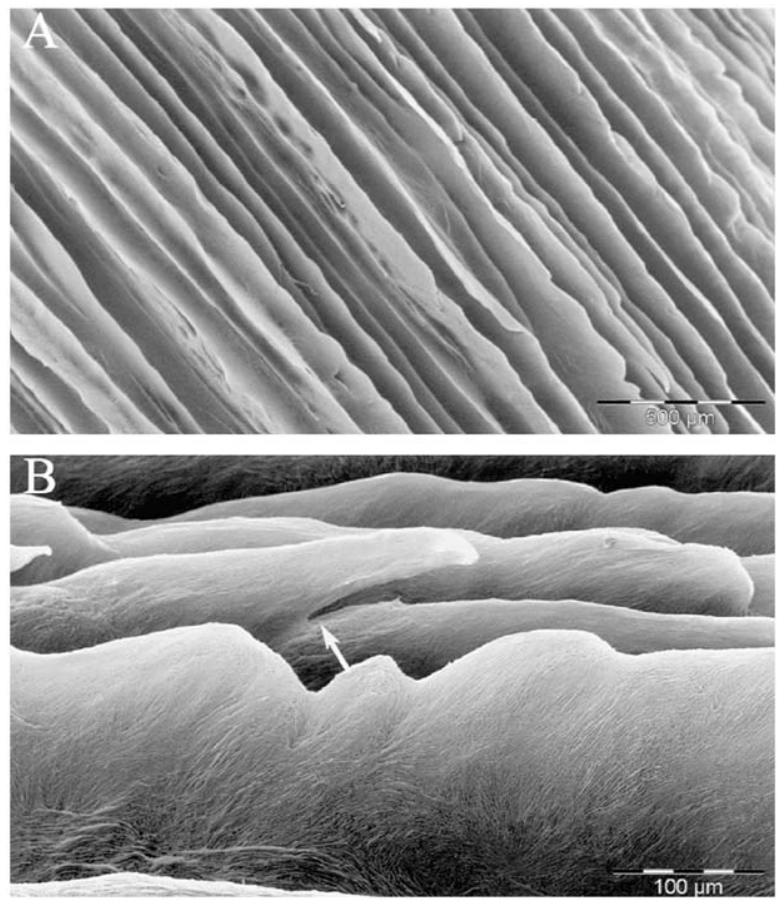

Figure 4. The nail bed in the visible nail is characterized by longitudinal trabecular dermal papillae $(\mathrm{A})$ with a free irregular margin: at this level, at high magnification, we can observe the incisions (arrow) due to nail growth forces (B). 
These loops gradually increase going towards the tip of the toe, while the angle they form with the axis of the crestae gradually decreasing.

The longitudinal trabeculae do not anastomize and at high magnification do not reveal any collagenous specialized structures such as exit holes of nerve fibers or oriented strands.

\section{The hyponychium}

At the end of the nail bed, when it turns into the dermal sheet of the digital tip, the longitudinal crestae become increasingly elongated constituting the dermal conic papillae of this area.

The region sited at the passage between the nail bed and finger tip, called Hyponychium, is characterized by the absence of dermal papillae and by a clear disorganization in the disposition of terminal crestae coming from the nail bed (Fig. 5A). In particular, this site is characterized by the presence of some bi or triheaded dermal papillae that
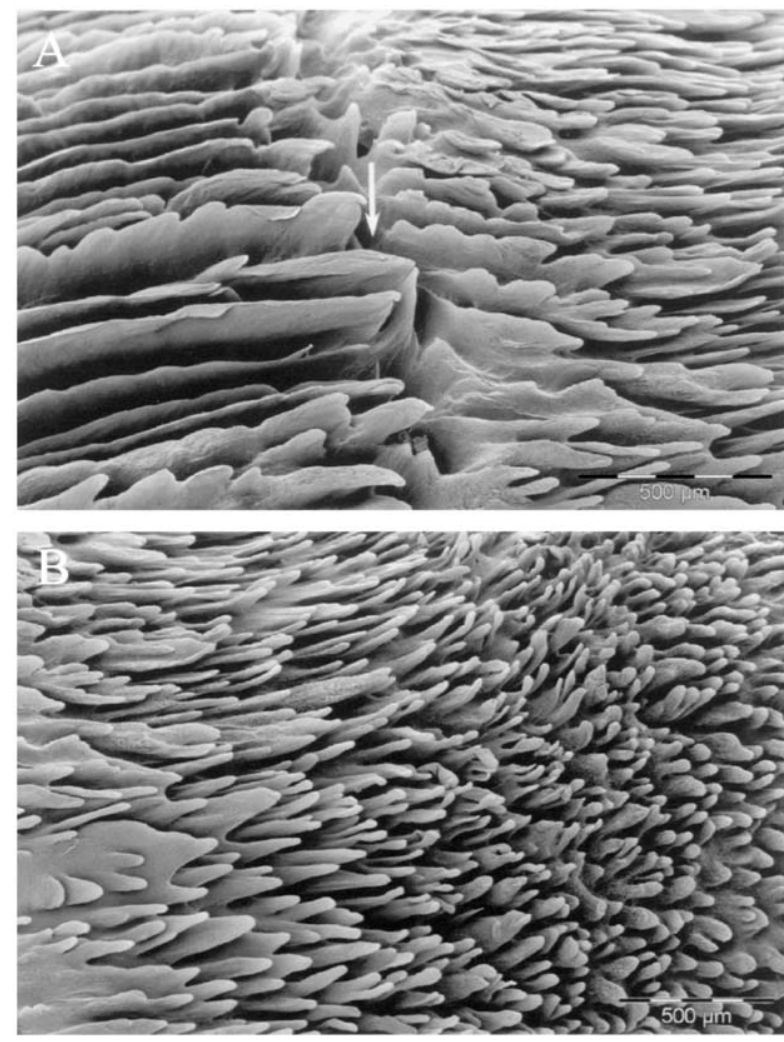

Figure 5. The hyponychium: note the passage from the trabecular longitudinal disposition of papillae to the elongated papillae belonging to the digital tip: between these two different dispositions a deep groove (arrow) without any dermal specialization can be observed $(A)$. The digital tip: note some two or three headed dermal papillae that very soon turn into other more elongated papillae that follow the natural convexity of the human digital tip (B). project their digitiform protrusions towards the tip of the toe.

\section{The tip of the toe}

The phalanged tip is characterized by high digitiform or filiform dermal papillae following the normal convexity of this region (Fig. 5B). The height of these structures increases towards the tip of the toe and decreases when the dorsal skin gradually turns into the plantar one characterized by toeprints. In this region we notice the highest density of dermal papillae, with an interpapillary distance of a few microns. The papillae are $100 \mu \mathrm{m}$ high and the diameter of their section measured at their origin is about $30 \mu \mathrm{m}$. At high magnification, exit holes of glands and some small holes related to free nerve fibers can be seen.

\section{The nail root}

The three-dimensional disposition of the nail root collagenous matrix varies frequently in the small space extending from the proximal edge of the nail plate to the visible nail.

For these reasons, it is useful to divide the nail root into three regions: proximal, median and distal. The proximal region is included between the nail matrix, where the proximal edge of the nail plate is embedded in a V-shaped epidermal invagination, and the beginning of dermal longitudinal trabecular papillae. In this area, very small dermal papillae constituting five or six series are transversally oriented to the longitudinal axis of the digit. The papillae appear as small peduncles measuring about $10 \mu \mathrm{m}$ in height and diameter (Fig. 6A).

The median region begins when the proximal series of dermal papillae gradually turn about $60^{\circ}$ along the nail bed giving rise to many longitudinal trabeculae parallel to one another. In the free margin of these trabeculae, same small dermal papillae that constitute the proximal region can be seen gradually elongating and following the natural growth direction of the nail plate (Fig. 6B). These projections sited on the free margin of the collagen trabeculae end in a single row of well-visible filiform collagenous strands. This imaginary line can be deemed the boundary between the medial region and the distal region.

The distal region is represented by the longitudinal trabeculae constituting the dermal papillae for all the nail bed, to the hyponychium. At the bottom of these trabeculae, we often find holes that penetrate the dermal layer constituting a 

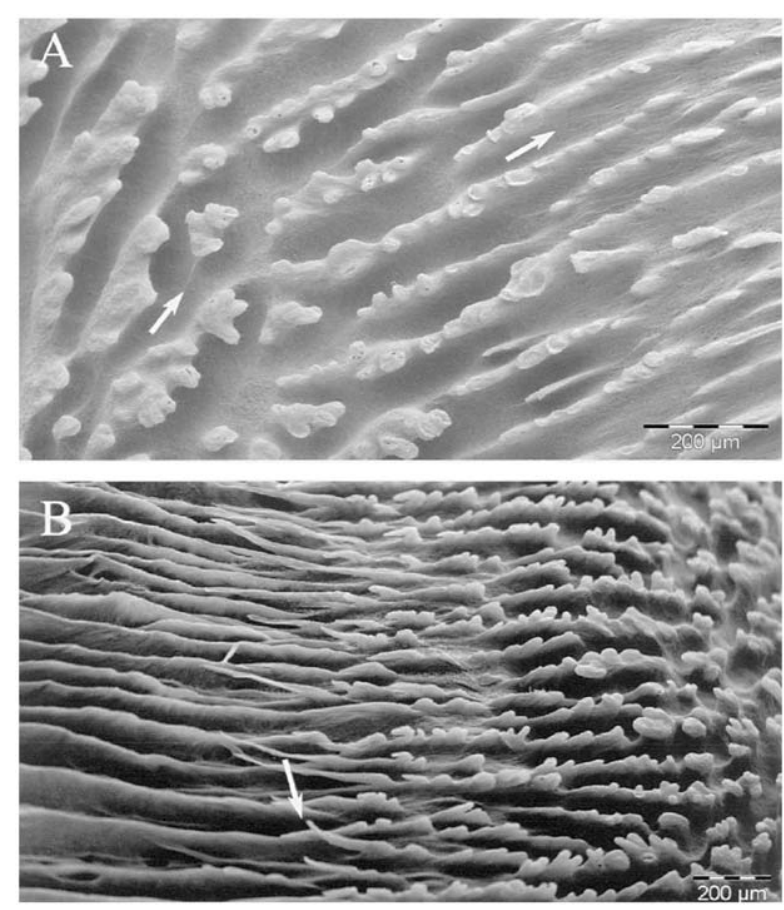

Figure 6. The proximal area of the nail root: we clearly see the small spherical dermal papillae arranged in three or four series transversally displaced, that gradually turn into the trabecular dermal papillae longitudinally oriented along the major axis of the digit (arrows) (A). An overview of the median area of the nail root. Note the longitudinal trabeculae with the irregular free margin occupied by small spherical dermal papillae that end in a series of filiform dermal papillae longitudinally oriented (arrow). This represents the limit between the median and distal parts of the nail root $(B)$.

tubular interconnected net under the plate (Fig. 7A/B): these holes are concentrated at the origin of the distal part where the trabeculae take on their usual form and disposition (Fig. 7C). At high magnification, some collagen plicae in the form of a "swallow's nest" can be seen attached to the trabecular walls (Fig. 7D).

\section{The perionychium}

The region between the lateral nail walls and the nail bed form a deep nail groove called the perionychium: in this area, the dermal papillae are very elongated and are stretched by the growing force of the nail.

At low magnification, a few dermal papillae are in contact with the nail border that gradually rises going towards the tip of the toe (Fig. 8).

The nail groove is characterized by the absence of dermal papillae and, at high magnification there is only a wide collagen sheet with no superficial specializations.

\section{The plantar skin}

The region of the plantar skin is characterized by the toeprints that we can clearly follow observing the disposition of dermal papillae (Fig. 9A). These papillae are arranged in regular rows corresponding to the visible superficial furrows of the plantar skin. The openings of excretory ducts of sweat glands are visible in the groove between two rows of dermal papillae (Fig. 9B).

At high magnification, the subtle collagenous structure of these ducts is made up of spiral collagenous continuous bundles forming a tubular empty structure that penetrates the dermal layer (Fig. 9C).

In a transverse section of the skin in the plantar region, we can clearly observe the longitudinal disposition of vessels distributed in two different layers projecting the capillary loops inside dermal papillae (Fig. 10A). Sometimes different corpuscles can be observed: thermoreceptors, mechanoreceptors, glomi, sweat glands and so on.

The tubular structures present in the thickness of the skin can be distinguished, since the collagenous structure of vessels varies from that of the tubules of sweat glands: observing the disposition of the collagenous strands in a vessel at high magnification, they are parallel to the major axis of the vessel, whereas in the tubules they assume a circular disposition, that becomes spiral near their superficial openings, as described before $(10 B / C)$.

\section{Discussion}

The human digit represents a specialized organ with tactile, stereognosic and thermic functions: the anatomical controller of its duties is the extracellular matrix that can be well visualized in three dimensions by Ohtani maceration and SEM analysis.

The collagen matrix of the digit plays a basic role in mechanical support and organization of all its components: it is an organized grid for epithelial cells, vessels and dermal corpuscles that constitute the whole thickness of digital skin (Omagari and Ogawa, 1990).

The collagenous tissue is always subjected to remodelling processes: in the digit it must also guarantee the growing process of the nail. In addition, the nail plate influences the disposition of collagenous structures of the dermal and hypodermal layers.

This is clearly visible in those regions where the pressure induced by nail growth on soft tissues is very high, such as in nail walls and the perionychium, where the dermal papillae are stretched by 

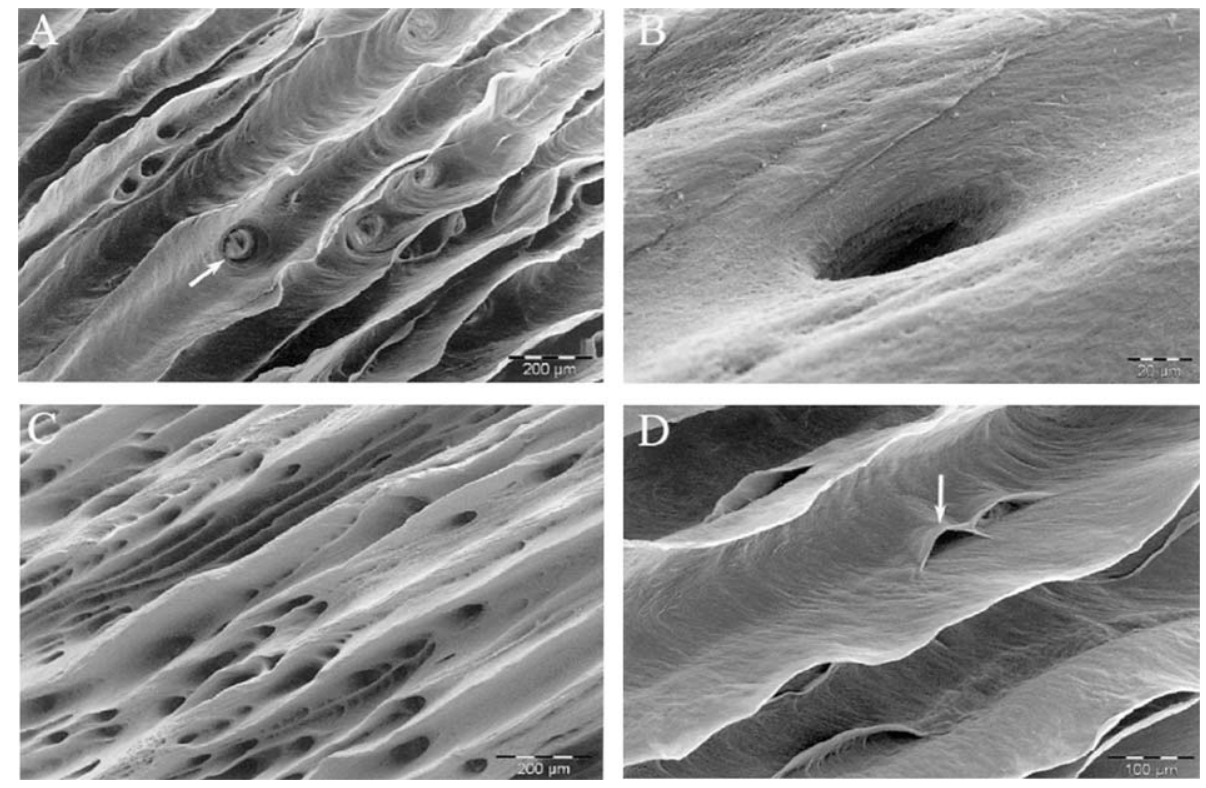

Figure 7. We can observe the holes (arrow) opening in the grooves formed between one trabecular papilla and the other (A). The high magnification reveals the three-dimensional shape of these holes (B). The highest concentration of these holes is visible at the beginning of dermal trabecular papillae (C). Attached to the wall of these trabeculae are collagen sheets in the form of a swallow's nest (arrow) (D).

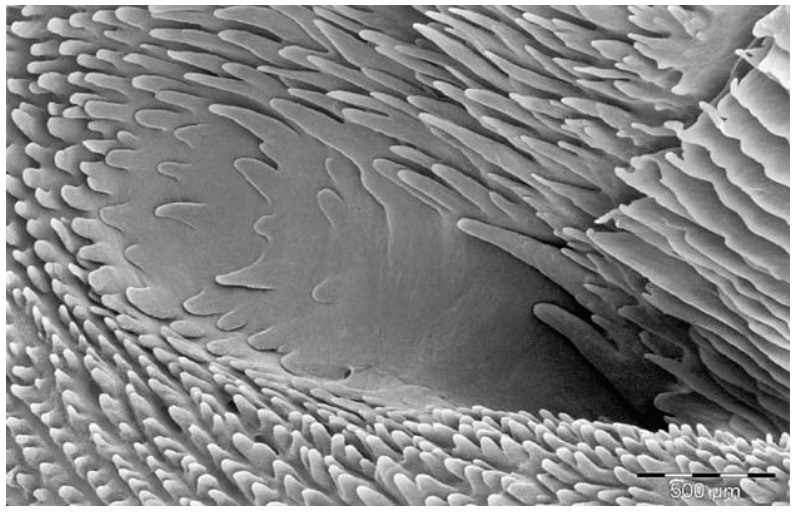

Figure 8. The peryonychium: note the stretched dermal papillae of the nail groove and their disposition on the nail wall and nail bed.

the shearing forces acting on them (Arao et al., 1998).

Traumatic injury to the finger tip and in particular to the hyponychium are well represented by the absence of the dermal papillae and their extremely irregular shape.

In the nail bed, the direction of dermal papillae follows the natural direction of nail growth that probably drags the subcutaneous tissues during its motion forming the characteristic irregular shape of the free margin observed on the collagenic trabeculae.

Moreover, the striae usually observed in the nail plate are probably due to the underlying
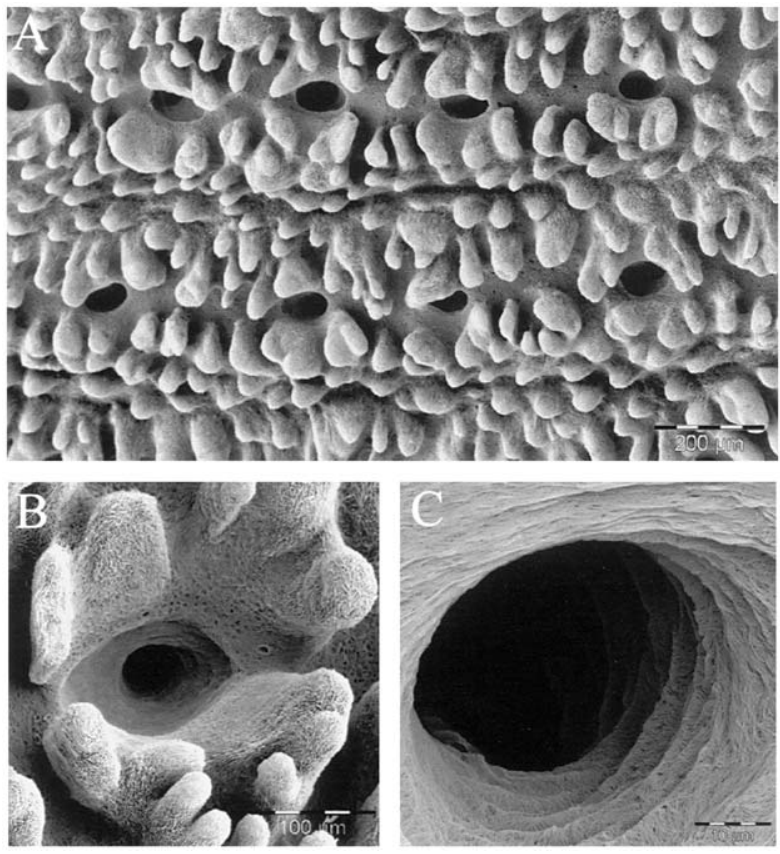

Figure 9. Plantar skin: the row-like disposition of dermal papillae drawing the toeprint scheme $(A)$ : the grooves between two rows contain a series of opening holes of the excretory ducts. High magnification of this hole (B). High magnification of the spiral collagenic bundles surrounding the empty cavity previously "filled" by an excretory duct $(C)$.

conformation of dermal papillae that are longitudinally oriented along the major axis of the digit. For these reasons, any change in this 

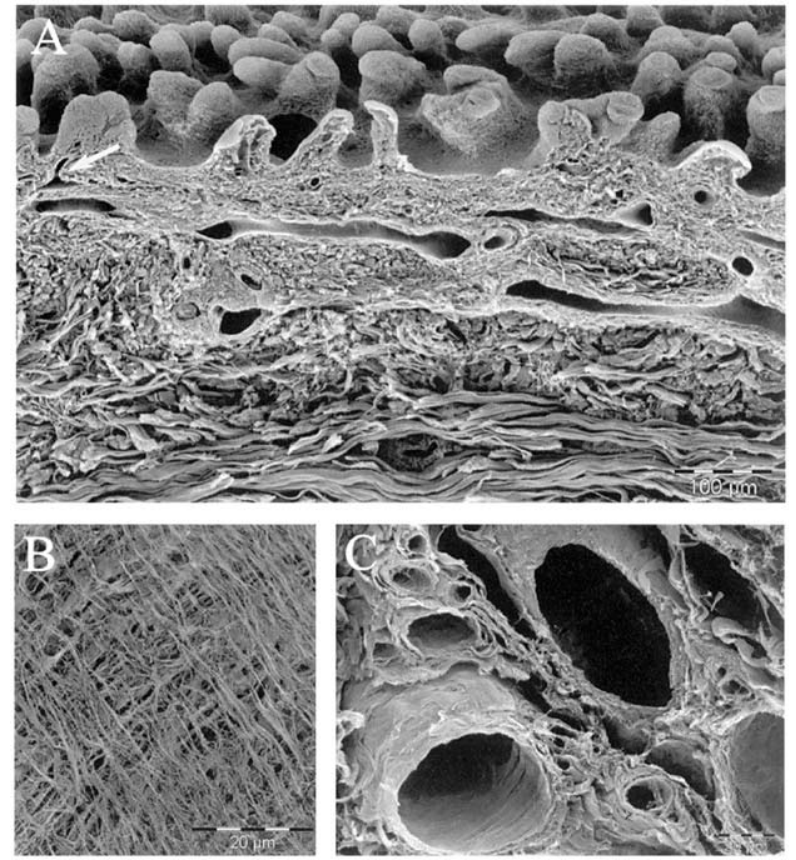

Figure 10. Transverse section of the plantar skin $(A)$ : note the vessels of the dermal layer longitudinally sectioned and the collagen bundles in the hypodermal layer. A vascular (B) structure can be distinguished from a tubular one $(C)$ observing the disposition of collagen bundles constituting their collagenic structure.

well-defined architecture may result in a visible modification of both the skin and the nail (Briggaman, 1982; Lavker et al., 1986, 1989).

Many pathological processes reducing or increasing the production of collagen fibers lead to changes in the morphology of the digit and impair its tactile, stereognosic and thermal functions. Connectivitis, congenital anomalies, ischemic processes or systemic diseases can induce many changes to the digits that we can try to explain starting from the collagenous architecture in the dermal layer.

Knowledge of the normal morphological shape that dermal papillae assume in the different regions of digital skin, enhances our understanding of the macroscopic changes occurring to the digit. For instance, one of the most important clinical signs of sclerodermia is sclerodactily leading to sausageshaped digits, while in chronic obstructive or interstitial pulmonary disease we can often observe "drum stick" fingers characterized by increased nail curvature and expansion of the distal phalanges.

At high magnification, the three-dimensional disposition of collagen bundles can be observed in the basement membrane and on the dermal papillae: the basement membrane resembled a honeycomb owing to the numerous compartments and recesses formed by the ridges and septae (Imayama, 1981; Pollitt, 1994; Yamada et al., 1996). The basal cells find an important attachment site in this disposition: they extend cytoplasmic processes into two or more compartments formed by the ridges and septae which considerably amplify the basement membrane surface available for epidermal attachment (Pierard, 1986).

Looking deeper into the thickness of the digital skin, we find a wide meshwork of tubular structures freely distributed in the dermal layer or organized in isolated structures such as sweat or sebaceous glands (Marcucci and Castano, 1990).

We can distinguish between the connective structure of medium-sized vessels and that of a tubule in sweat glands by looking at the orientation of the collagen fibers in their innermost layers. The longitudinal orientation of collagenous bundles is characteristic of the innermost layer of the intimal sheet in vessels while the circular or spiral disposition is characteristic of tubules in sweat and sebaceous glands. The spiral disposition of these collagenous bundles demonstrates the original mechanism of formation of sweat and sebaceous glands that enter the dermal layer like a corkscrew: the torsion forces acting on fibrillogenesis could underlie this three-dimensional disposition (Dell and Munger, 1986). Moreover, it is wellknown how the myocytes in close contact with the collagenous matrix are spirally displaced around the excretory tubules of these glands to allow a correct excretory function.

The gradual reduction of the thickness of the dermal layer in the dorsal digital skin going towards the eponychium is also demonstrated by a decrease of the number of exit holes for sweat and sebaceous glands. The same process is observed for the small holes of free nervous epidermal fibers that gradually disappear in the free margin of the eponychium (Cauna, 1980).

The nail root is one of the most important sites of ongoing tissue regeneration processes. Physiological nail growth is based on the possibility of connective, vascular and epidermal tissue to provide the continued organized cell turnover. The support for these processes is guaranteed by the dermal extracellular matrix that in the nail root assumes a particular disposition in the form of its dermal papillae. The passage from one disposition to another is clearly revealed by a change in the direction and shape of dermal papillae.

We have also found some unusual dermal papillae shapes, in particular, in the nail root where the stress forces and the mechanism of fibrillogenesis causes ongoing collagenic remodelling. This is mostly visible in the junction between the median and distal parts of 
the nail root, when the free margin of trabecular papillae forms a series of filiform collagenic protrusions parallel to the longitudinal lining of the nail bed but probably with aspecific duties. Due to their dimensions, these papillae do not contain any vascular support, as demonstrated by the corrosion-casting technique applied to human digit.

The vascular three-dimensional disposition of capillaries in all the regions studied corresponded exactly to the shape of the dermal papillae in which they were "enclosed". The capillary loops observed in dorsal skin and tip of the phalangs were similar in size to those observed in the dermal papillae of the same regions. The effect of the stretching forces acting on the perionychium that causes a distortion and absence of dermal papillae in the nail groove is well visible also in its vascular structure, made up of a wide net of capillaries. The peculiar disposition of dermal papillae under the visible nail is comparable to the capillary loops observed in the same region, as for the nail root where small spherical papillae are visible on the vascular cast as regular bulges. In this region, the holes in the grooves between one trabecular papilla and the other are new findings but the maceration did not disclose their origin. For this reason, work is still underway to understand their nature by serial light microscopic section.

The impressive correspondence between the vascular structure and dermal papillae suggests a clear cut close relationship in the formation and modification of collagenous structure in human digit through its vascular support (Imayama, 1981; Umeda and Ikeda, 1998; Hagisawa et al., 2001). This fact may underlie many pathophysiological conditions in which an alteration in blood supply can impair collagenic turnover with consequent morphological modifications of dermal papillae.

\section{References}

Arao, H., Obata, M., Shimada, T., Hagisawa, S., 1998. Morphological characteristics of the dermal papillae in the development of pressure sores. J. Tissue Viability 8, 17-23.

Bolanowski, S.J., Pawson, L., 2003. Organization of Meissner corpuscles in the glabrous skin of monkey and cat. Somatosens Mot. Res. 20, 223-231.

Briggaman, R.A., 1982. Epidermal-dermal interactions in adult skin. J. Invest. Dermatol. 79, 21s-24s.

Cauna, N., 1980. Fine morphological characteristics and microtopography of the free nerve endings of the human digital skin. Anat. Rec. 198, 643-656.

Dell, D.A., Munger, B.L., 1986. The early embryogenesis of papillary (sweat duct) ridges in primate glabrous skin: the dermatotopic map of cutaneous mechanoreceptors and dermatoglyphics. J. Comp. Neurol. 244, 511-532.

Hagisawa, S., Shimada, T., Arao, H., Asada, Y., 2001. Morphological architecture and distribution of blood capillaries and elastic fibres in the human skin. J. Tissue Viability 11, 59-63.

Imayama, S., 1981. Scanning and transmission electron microscope study on the terminal blood vessels of the rat skin. J. Invest. Dermatol. 76, 151-157.

Kitano, Y., Okada, N., 1983. Separation of the epidermal sheet by dispase. Br. J. Dermatol. 108, 555-560.

Lavker, R.M., Zheng, P.S., Dong, G., 1986. Morphology of aged skin. Dermatol. Clin. 4, 379-389.

Lavker, R.M., Zheng, P.S., Dong, G., 1989. Morphology of aged skin. Clin. Geriatr. Med. 5, 53-67.

Marcucci, A., Castano, P., 1990. Eccrine sweat glands of rat fingertips. Scanning electron microscope observations after enzymatic digestion of dermal connective tissue. Acta Anat. 137, 31-36.

Moore, J.C., Ray, A.K., Shakespeare, P.G., 1993. Surface ultrastructure of human dermis and wounds. Br. J. Plast. Surg. 46, 460-465.

Ohtani, O., Ohtani, Y., 2000. The maceration technique in scanning electron microscopy of collagen fiber frameworks: its application in the study of human livers. Arch. Histol. Cytol. 55, 225-232.

Omagari, N., Ogawa, K., 1990. Three dimensional arrangement of fibrocytes in the dermal papilla of the human sole skin. Okajimas Folia Anat. Japan 67, 195-202.

Pierard, G.E., 1986. How is the basement membrane attached to the dermis? Stereology of the interlacing fibers at the underface of the dermo epidermal basement membrane. Am. J. Dermopathol. 8, 234-236.

Pollitt, C.C., 1994. The basement membrane at the equine hoof dermal epidermal junction. Equine Vet. J. 26, 399-407.

Reed, C.C., lozzo, R.V., 2002. The role of decorin in collagen fibrillogenesis and skin homeostasis. Glycoconj. J. 19, 249-255.

Sangiorgi, S., Manelli, A., Congiu, T., Bini, A., Pilato, G., Reguzzoni, M., Raspanti, M., 2004. Microvascularization of the human digit as studied by corrosion casting. J. Anat. 204, 123-131.

Torre, C., Varetto, L., 1985. SEM study of dermal surface. A new approach to forensic traumatology. Am. J. Forensic Med. Pathol. 6, 256-270.

Torre, C., Varetto, L., Mattutino, G., 1986. Dermal surface morphology in wound healing. An experimental scanning electron microscope study. Am. J. Forensic Med. Pathol. 7, 337-343.

Tsugane, M., Yasuda, M., 1995. Dermatoglyphics on volar skin of mice: the normal pattern. Anat. Rec. 242, 225-232.

Tsuji, T., 1980. Elastic fibres in dermal papilla. Scanning and transmission electron microscopic studies. Br. J. Dermatol. 102, 413-417.

Umeda, N., Ikeda, A., 1998. Scanning electron microscopic study of the capillary loops in the dermal 
papillae. Skin of the hand of the Japanese monkey (Macaca fuscata). Acta Anat. 132, 270-275.

Vaccaro, M., Pergolizzi, S., Mondello, M.R., Santoro, G., Cannavo, S.P., Guarnieri, B., et al., 1999. The dermoepidermal junction in psoriatic skin as revealed by scanning electron microscopy. Arch. Dermatol. Res. 291, 396-399.

Warfel, K.A., Hull, M.T., 1989. Ultrastructure of the epidermal-dermal junction and cutaneous basal lamina in Bowen's disease. J. Cutan. Pathol. 16, 203-206.

Wilborn, W.H., Montes, L.F., 1979. Scanning electron microscopy of dermatofibromas. J. Cutan. Pathol. 6, 323-328.

Yamada, N., Kashima, Y., Inoue, T., 1996. Scanning electron microscopy of the basal surface of the epidermis of human digits. Acta Anat. 155, 242-248. 